\title{
ESTIMULAÇÃO COGNITIVA PARA MELHORIA DAS FUNÇÕES CEREBRAIS DE IDOSOS
}

\author{
Adriana Schüler Cavalli \\ Universidade Federal de Pelotas \\ adriscavalli@gmail.com \\ Gabriel Guadalupe Barcelos Oliveira \\ Universidade Federal de Pelotas \\ gabrielguadalupeoleveira@gmail.com
}

\author{
Giovana Duzzo Gamaro \\ Universidade Federal de Pelotas \\ giovanagamaro@hotmail.com
}

Karoline Da Silva Duarte Universidade Federal de Pelotas

karolinedsd@hotmail.com

Marcelo Olivera Cavalli Universidade Federal de Pelotas maltcavalli@gmail.com

\begin{abstract}
Resumo
A memória é um dos aspectos mais importantes da neurociência, principalmente no que concerne aos processos de ensino e de aprendizagem. Atividades que requerem atenção e pensamento lógico aumentam a densidade sináptica cerebral melhorando o desempenho mnemônico. A Universidade Federal de Pelotas/Rio Grande do Sul oferece aos idosos da comunidade a Oficina de Memória e Estimulação Cognitiva com aulas semanais de 60 minutos. O objetivo deste estudo foi analisar a motivação dos idosos ao executarem as atividades propostas nas aulas. Para tanto foi utilizado o Inventário de Motivação Intrínseca. Participaram do estudo 24 idosos, sendo observado que 83,3\% destes afirmaram que as aulas eram "Muito divertidas"; 75\% demonstraram estar muito satisfeitos com seu desempenho nas atividades; 91,6\% afirmaram ter se esforçado bastante no cumprimento das atividades. Os resultados demonstraram que os idosos estavam motivados na realização das aulas de estimulação cognitiva.
\end{abstract}

Palavras-chave: Atividade Motora. Cognição. Ensino. Idoso. Memória.

\section{COGNITIVE STIMULATION FOR IMPROVING CEREBRAL FUNCTIONS OF ELDERLY}

\begin{abstract}
Memory is one of the most important aspects of neuroscience, especially regarding teaching and learning processes. Activities that require attention and logical thinking increase brain synaptic density by improving the mnemonic performance. The Federal University of Pelotas/RS offers to the elderly of the community 60-minute weekly classes in the Workshop of Memory and Cognitive Stimulation. The objective of this study was to analyze the motivation of the elderly when performing the activities proposed in the classes. For that, the Intrinsic Motivation Inventory was used. Twenty-four elderly people participated in the study, and $83.3 \%$ affirmed that the classes were "Very fun"; $75 \%$ were very satisfied with their performance in the activities; $91.6 \%$ stated that they had made considerable effort to carry out their activities. The results indicate that the elderly were motivated to take classes of cognitive stimulation.
\end{abstract}

Keywords: Motor Activity. Cognition. Education. Aged. Memory.

\section{ESTIMULACIÓN COGNITIVA PARA MEJORA DE LAS FUNCIONES CEREBRALES DE ANCIANOS}

\section{Resumen}

La memoria es uno de los aspectos más importantes de la neurociencia, principalmente en lo que concierne a los procesos de enseñanza y aprendizaje. Las actividades que requieren atención y pensamiento lógico aumentan la densidad sináptica cerebral mejorando el rendimiento mnemónico. La Universidad Federal de Pelotas/RS ofrece a los ancianos de la comunidad el Taller de Memoria y Estimulación Cognitiva con lecciones semanales de 60 minutos. El objetivo de este estudio fue analizar la motivación de los ancianos al ejecutar las actividades propuestas en las clases. Para ello se utilizó el Inventario de Motivación Intrínseca. Participaron del estudio 24 ancianos, siendo observado que el 83,3\% de éstos afirmaron que la clase era "Muy divertida"; 75\% demostraron estar muy satisfechos con su desempeño en las actividades; el 91,6\% afirmó haberse esforzado bastante en el cumplimiento de las actividades. Los resultados demostraron que los ancianos estaban motivados en la realización de las clases de estimulación cognitiva.

Palavras clave: Actividad Motora. Cognición. Educación. Anciano. Memoria. 
Estimulação cognitiva para melhoria das funções cerebrais de idosos

\section{INTRODUÇÃO}

Segundo estimativa do Instituto Brasileiro de Geografia e Estatística (IBGE, 2010), próximo a 18,7\% da população brasileira será idosa em 2030. Levando em consideração as perdas das capacidades funcionais e cognitivas decorrentes do processo de envelhecimento (IZQUIERDO, 2011), a demanda de atendimento especializado para idosos tende a aumentar nos próximos anos. Do ponto de vista epidemiológico, a demência vem rapidamente se tornando uma questão de saúde pública, visto que a prevalência da doença no Brasil é semelhante aos países desenvolvidos (SCHMIDT et al., 2011).

Demência pode ser definida como uma síndrome clínica caracterizada por sinais de dificuldades de memória, distúrbios na linguagem e em outras funções cognitivas, mudanças na conduta e dificuldades na execução das atividades de vida diária (QIU, KIVIPELTO, STRAUSS, 2009).

Nesse sentido, os indivíduos idosos naturalmente demonstram preocupação com o declínio cognitivo fisiológico para a demência e lamentam a diminuição do seu desempenho mnemônico (SOUZA e CHAVES, 2005).

Sendo assim, este estudo tem como objetivo avaliar a motivação dos participantes para a execução das atividades propostas na Oficina de Memória e Estimulação Cognitiva (OMEC) oferecida no Projeto Núcleo de Atividades para a Terceira Idade (NATI) vinculado à Escola Superior de Educação Física da Universidade Federal de Pelotas/RS (ESEF/UFPel/RS). A OMEC tem como objetivo o desenvolvimento de diferentes estratégias voltadas à manutenção e melhoramento do desempenho da memória de idosos.

\section{MATERIAL E MÉTODOS}

O estudo tem um delineamento transversal descritivo (THOMAS, NELSON, 2002).

A amostra foi intencional e composta por 24 idosos participantes da OMEC. Os idosos devidamente matriculados na OMEC no segundo semestre de 2016 foram convidados a participar do estudo e assinaram o Termo de Consentimento Livre e Esclarecido.

As aulas foram ofertadas uma vez por semana com duração de 60 minutos. Inicialmente foram realizados alongamentos, seguidos de atividades de leitura de pequenos textos e posterior interpretação. Após esses, foram realizados jogos que estimularam os sentidos, a atenção e a concentração. 
Estimulação cognitiva para melhoria das funções cerebrais de idosos

A OMEC é oferecida semestralmente para pessoas com 60 anos ou mais e consiste em 15 encontros. Para coleta dos dados foi utilizado o Inventário de Motivação Intrínseca (IMI) de Mcauley, Duncan e Tammen (1989), que é um instrumento que permite avaliar a intensidade da motivação intrínseca dos indivíduos em qualquer atividade, através de 9 questões com respostas definidas em uma escala Likert de 7 pontos, com respostas desde "isso me motiva pouquíssimo" a "isso me motiva muitíssimo". O IMI procura identificar o quão satisfeito o indivíduo está na realização das tarefas propostas, questionando competência, desempenho e realização. Este instrumento foi aplicado ao final da $14^{\circ}$ encontro, ou seja, penúltimo dia de aula. Os dados foram analisados em valores absolutos e percentuais.

\section{RESULTADOS E ANÁLISE}

Participaram do estudo todos os 24 idosos participantes da OMEC. A amostra foi composta por 22 mulheres (73,81 \pm 7,55 anos) e dois homens ( $69 \pm 11,3$ anos).

Com relação à motivação dos sujeitos para a realização das atividades, 83,3\% dos idosos afirmaram a aula ser "Muito divertida"; 87,5\% relataram que as aulas eram "Nada Chata"; 54,1\% definiram se sentirem "Muito competentes" após as aulas; 75\% estavam muito satisfeitos com seu desempenho; 91,6\% afirmaram ter se esforçado bastante nas atividades propostas e 95,8\% relataram que era "Muito importante" desempenhar bem as atividades das aulas, conforme Tabela 1 abaixo.

De acordo com Yassuda (2002), muitos estudos indicam que intervenções envolvendo técnicas de memorização, relaxamento e atenção, podem gerar efeitos positivos e duradouros em idosos, especialmente quando empregadas em grupo.

Ainda, segundo Souza e Chaves (2005), a execução de exercícios de estimulação cognitiva através de atividades que requeiram atenção e pensamento lógico é recomendada, pois os mesmos contribuem para o aumento da densidade sináptica cerebral.

Segundo dados de Izquierdo et al. (2013), o melhor exercício para manter a memória é a leitura, pois ao fazer isto, as memórias visuais e verbais entram em funcionamento.

Quanto a motivação em participar das atividades educativas propostas, García (2009, citado por CACHIONI et al., 2015, p. 97) ressalta que "Aprende-se melhor e mais depressa se houver interesse pelo assunto. Motivado, o idoso possui uma atitude ativa e empenhada no processo de aprendizagem e, por isso, aprende melhor". Os idosos da OMEC demonstraram estar motivados pelas atividades desenvolvidas nas aulas evidenciando que a metodologia 
Estimulação cognitiva para melhoria das funções cerebrais de idosos

empregada parece estar ao encontro do interesse dos alunos e, portanto, devendo ser considerada para a melhoria e estimulação das habilidades cognitivas dos idosos.

Ainda referenciando García (2009, citado por CACHIONI et al., 2015, p. 97), o mesmo salienta que "Quanto mais diversificadas forem as abordagens de um tema, quanto mais diferenciadas as tarefas, maior é a motivação e a concentração e melhor decorre a aprendizagem". As atividades na OMEC através de diferentes tipos de jogos (de atenção, de concentração e sensoriais), além da leitura de textos sobre diversos temas, possibilitando pequenos debates com base nas experiências e vivências dos idosos, parecem corroborar com a ideia de que a escolha metodológica das aulas está cumprindo seu propósito e auxiliando seus participantes, os idosos.

Tabela 1 - Inventário de Motivação Intrínseca dos participantes da OMEC.

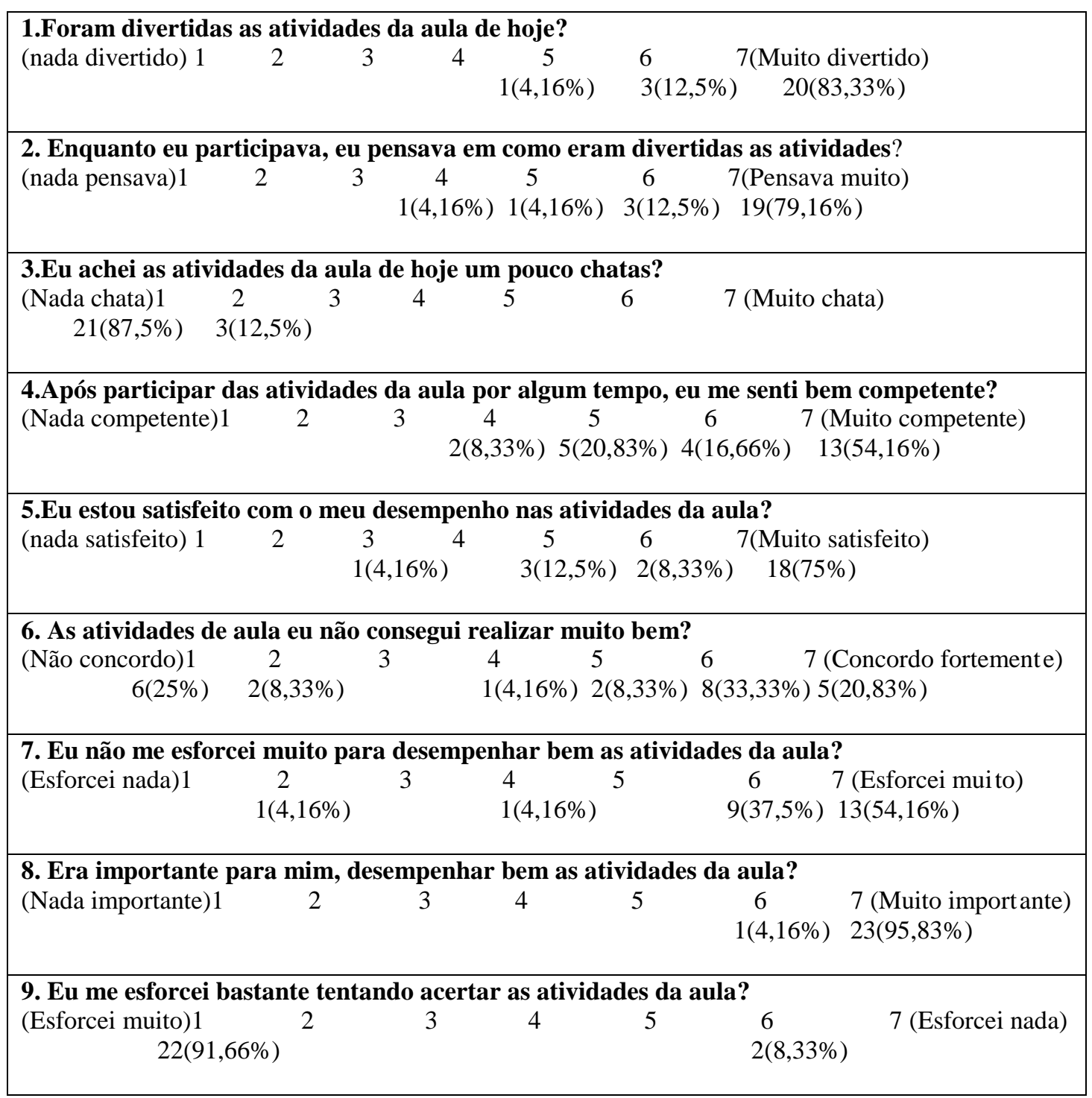




\section{CONSIDERAÇÕES FINAIS}

Os resultados demonstraram que os idosos estavam motivados e gostaram de participar das aulas. São necessários mais estudos na área para um melhor entendimento de como a motivação pode permitir a adesão e permanência dos idosos em projetos, assim como, para compreender como a dinâmica das aulas pode auxiliar na criação de modelos de orientação adequados para a população idosa.

\section{REFERÊNCIAS}

CACHIONI, M.; ORDONEZ, T.N.; BATISTONI, S.S.T.; LIMA-SILVA, T.B. Metodologias e estratégias pedagógicas utilizadas por educadores de uma Universidade Aberta à Terceira Idade. Educação \& Realidade, Porto Alegre, v.40, n.1, p.81-103. Jan./mar. 2015.

IBGE. Dados preliminares do Censo 2010 já revelam mudanças na pirâmide etária brasileira. Comunicação Social, 2010. Disponível em: $<$ http://www.ibge.gov.br/home/presidencia/noticias/noticia_visualiza.php?id_noticia=1722\&id _pagina=1>. Acesso em abril 2017.

IZQUIERDO, I. Memória. Porto Alegre: Artmed, 2011.

IZQUIERDO, et al. Memória: Tipos e Mecanismos - achados recentes. Revista USP. São Paulo, n.98, p.9-16. 2013.

MCAUlEY, E.; DUNCAN, T.; \& TAMMEN, V.V. Psychometric properties of the Intrinsic Motivation Inventory in a competitive sport setting: A confirmatory factor analysis. Research Quarterly for Exercise and Sport, 60: 48-58. 1989.

SCHMIDT et al. Health in Brazil 4. Chronic non-communicable diseases in Brazil: burden and current challenges. The Lancet, 377:1949-61. May, 2011. Disponível em< http.// www.thelancet.com. Acesso em junho 2017.

SOUZA, J.N.; CHAVES, E.C. O efeito do exercício de estimulação da memória em idosos saudáveis. Rev Esc Enferm USP. 39 (1):13-9. 2005.

THOMAS, J.R.; NELSON, J.K. Métodos de pesquisa em atividade física. $3^{\mathrm{a} e d .}$ Porto Alegre, Artmed Editora, 2002.

QIU, C.; KIVIPELTO, M.; STRAUSS, E. Epidemiology of Alzheimer's disease: occurrence, determinants, and strategies toward intervention. Dialogues Clin Neurosci. 11:111-128. 2009. 
Estimulação cognitiva para melhoria das funções cerebrais de idosos

YASSUDA, M.S. Memória e envelhecimento saudável. In: FREITAS, E.V.; PY, L.; NERI, A.L.; CANÇADO, F.A.X.; GORZONI, M.; ROCHA, S.M. (Orgs.). Tratado de Geriatria e Gerontologia. Rio de Janeiro: Guanabara Koogan, p.914-9. 2002. 